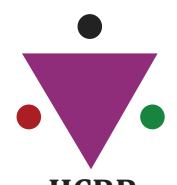

IJCRR

Section: Healthcare ISI Impact Factor (2019-20): 1.628 IC Value (2019): 90.81 $\operatorname{SJIF}(2020)=7.893$

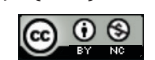

Copyright@IJCRR

\title{
"Single Nucleotide Polymorphisms of ATP-Binding Cassette Gene(ABCC3 rs4793665) affect High Dose Methotrexate-Induced Nephrotoxicity in Children with Osteosarcoma"
}

\author{
Amr Y. Zakaria1, Rania M. Labib², Sameh A. Abdelshafi ${ }^{3}$, Asmaa Salama4, \\ Omneya Hassanain ${ }^{5}$, Hoda Rabea ${ }^{6}$, Enas El Nadi ${ }^{7}$
}

\begin{abstract}
'Pharm D, Faculty of Pharmacy Beni-Suef University, Teaching Assistant Clinical Pharmacy (Pharmacy Practice) Department, Faculty of Pharmacy, Horus University (HUE), New Damietta, Egypt; ${ }^{2}$ PhD, Research Department, Children's Cancer Hospital-Egypt-57357, Cairo,

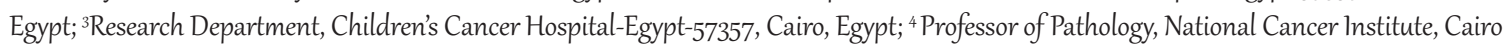
University, Consultant of Pathology, Children's Cancer Hospital-Egypt-57357, Cairo, Egypt; ${ }^{5}$ Epidemiology and Biostatistics Unit, Research Department, Children's Cancer Hospital-Egypt-57357, Cairo, Egypt; ${ }^{6} \mathrm{PhD}$, Clinical Pharmacy Department, Faculty of Pharmacy Beni-Suef University, Egypt; ${ }^{7}$ Assistant Professor Medical Oncology Department Faculty of Medicine Beni-Suef University, Consultant of Pediatric Oncology, Children's Cancer Hospital-Egypt-57357, Cairo, Egypt.
\end{abstract}

\section{ABSTRACT}

Background: Osteosarcoma (OS) is one of the aggressive bone tumours commonly diagnosed in adolescents and young adults.

Aim: The study aimed to investigate the effect of 9 single nucleotide polymorphisms (SNPs) in 5 genes on methotrexate (MTX) plasma level and the association between the SNPs and MTX toxicities.

Subjects and Methods: One hundred and thirty-three children with OS were enrolled and treated with four weeks of highdose methotrexate (HDMTX) as neo-adjuvant therapy in a children's cancer hospital. Blood samples were genotyped for the studied SNPs (MTHFR rs1801133C>T, SLC01B1 rs11045879C>T, rs4149081 A>G, rs2306283 A>G, ABCC3 rs4793665C>T, rs4148412T>C, rs733392G $>A$, ABCG2 rs2231142 G>T, and ABCC2 rs717620C>T using TaqMan ${ }^{\circledR}$ RT-PCR assay. Plasma concentrations of MTX were measured. Toxicities were evaluated, besides the studying of survival analysis and tumour necrosis (TN\%). Significance was considered at $p<0.004$.

Results: Older patients experienced an increased risk of delayed MTX elimination at 72-hour [ $p=0.00059, \mathrm{OR} 1.19,95 \% \mathrm{Cl}$ (1.09-1.29)]. Moreover, older patients were associated with protection of developing higher grades of enzymatic hepatotoxicity for $(\mathrm{ALT})[p=0.002$, OR $0.88,95 \% \mathrm{Cl}(0.8-0.95)]$ and $(\mathrm{AST})[p=0.001$, OR $0.82,95 \% \mathrm{Cl}(0.76-0.89)]$. Patients with $(C C)$ genotypegroup of $A B C C 3$ (rs4793665) $\mathrm{C}>\mathrm{T}(14 \%$ of the studied population) associated with protective effect against severe nephrotoxicity $[p=0.003$, OR $0.31,95 \% \mathrm{Cl}(0.14-0.67)]$.

Conclusion: The genotyping of $A B C C 3$ ( $r$ 4793665 C>T) and age could help in predicting patients with OS at risk of toxicities due to HDMTX.

Keywords: Osteosarcoma, SNPs, Methotrexate, Toxicity, Genotyping, ABCC3 (rs4793665).

\section{INTRODUCTION}

Osteosarcoma is one of the primary aggressive bone tumours most often diagnosed with approximately $70 \%$ in children and adolescents. The most frequent primary sites are the distal femur and proximal tibia. It is distinguished by mesenchymal spindle cells that deposit immature osteoid matrix. ${ }^{1}$ The Rizzoli Institute studies outlined a crucial role of MTX, cisplatin, and doxorubicin as the most active chemotherapeutic agents for OS. The study by El-Refaey et al. highlighted the evidence of 3-year disease-free survival rates of $60-70 \%$ following the administration of pre-operative multi-agent chemotherapy followed by surgical resection and maintenance chemotherapy. ${ }^{2}$ The European and American osteosarcoma study group (EURAMOS) protocol is an international randomized study on patients with OS,

\section{Corresponding Author:}

Rania M. Labib, Research Department, Children's Cancer Hospital., 1 El-Emam Road, Sayeda Zeinab, Cairo, Egypt; Tel:+201127744748; +20225351500 extension: 7205; Fax:+20223619036; Email: raniamlabib@gmail.com, amryahia645@gmail.com

ISSN: 2231-2196 (Print)

Received: 04.07 .2021
ISSN: 0975-5241 (Online)

Revised: 14.08 .2021
Accepted: 16.09 .2021
Published: 11.10 .2021 
which consists of HDMTX $\left(12 \mathrm{~g} / \mathrm{m}^{2}\right)$ infused over 4-hours per week as neo-adjuvant chemotherapy for four weeks. ${ }^{3}$ MTX is an antineoplastic agent and is prescribed in various cancer treatment protocols such as acute leukaemia, breast cancer, and OS. MTX competitively inhibits certain folatedependent enzymes and the biosynthesis of DNA nucleotides such as purines, pyrimidines, and encoded proteins resulting in apoptosis. ${ }^{4}$ Extracellular MTX is transported to the cell by various pathways, primarily through active transport mediated by reduced folate carriers. Passive diffusion adds to the transfer of MTX by cellular inflow. The majority of MTX is eliminated by renal excretion and less than $20 \%$ undergoes biliary secretion. MTX is transmitted intracellularly by the solute carrier organic anion transporters family member 1B1 (SLCO1B1). ${ }^{5}$ On the other hand, ABC genes that encode pglycoprotein are responsible for the efflux of MTX out of the cell, such as human multidrug resistance-related protein 2 (MRP 2), encoded by ABC subfamily C member2 (ABCC2). ${ }^{6}$ Methylene-tetrahydrofolate Reductase (MTHFR) 677C $>T$ rs 1801133 is located at the coding region of the gene and may affect its phenotype. Several studies thus far have linked this SNP with toxicity and clinical outcome. ${ }^{7}$ The useful PKs biomarker is the beta elimination half-life in the prediction of delayed elimination when extended. ${ }^{8}$

Substantially, HDMTX is included in many protocol treatments. HDMTX has a narrow therapeutic index. It can cause significant toxicities such as haematological toxicity, hepatotoxicity, and nephrotoxicity. Acute kidney injury (AKI) represents $2-12 \%$ of patients. Nephrotoxicity may be due to the MTX crystals in the lumen of the renal tubules, causing irreversible tubular toxicity. AKI and other toxicities of HDMTX can lead to significant treatment delaying, a decrease in renal function, and morbidity. ${ }^{9}$

Age, gender, and inter-individual genetic variations may be associated with a decrease in the effectiveness of drug therapy, or side effects, or toxicity, or treatment failure. ${ }^{10}$ According to the American Society of Health-System Pharmacists (ASHP), "Pharmacogenetics (PGs) investigates the effect of genomic variations on drug responses. PGs testing can improve drug response that may include sub-optimal clinical outcomes, decreased cost of treatment, better medication adherence, more appropriate selection of therapeutic agents, decreased length of treatment, and enhanced patient safety. " SNPs are the leading cause of genetic variations and account for approximately $90 \%$ of the variation in the human genome. It may influence the role or expression of the gene, the inter-individual differences in pharmacokinetics (PKs), and pharmacodynamics (PDs) parameters. ${ }^{11}$ Based on data available from the Pharmacogenomics Knowledge database (www.pharmagkb.com), we selected SNPs with a reported minor allele frequency higher than $10 \%(\mathrm{MAF} \geq 0.1)$ in the Caucasian (Egyptian populations). Each SNP of these genes is predicted to affect therapeutic limitation and clinical de- cisions, including ethnic PKs and PDs gene variations that affect phenotype. ${ }^{12}$ The current study aimed to determine the allelic frequencies of 9 SNPs in 5 genes (MTHFR, SLCO1B1, $A B C C 3, A B C G 2$, and $A B C C 2$ ) involved in the MTX pathway in the children with OS. Our study investigated the role of PGs variations on MTX elimination, toxicities, survival, and tumour necrosis (TN\%). Hopefully, this will help us in the future to build models that accurately predict strategies for individual patients and use this knowledge to personalize treatment plans.

\section{PATIENTS/METHODS}

\section{Design and participants:}

A retrospective cohort study included 133 patients diagnosed with a localized non-metastatic OS of the extremity treated based on the EURAMOS protocol at the hospital between 2013 and 2018 in Cairo, Egypt. The study was approved by the Institutional Review Board (IRB) with serial number 1112018 (25 June 2018). All patients and their parents or legal guardians have signed written informed consent. The samples and data were collected and documented between April 2018 to Jan 2019. Patients were included if they fit the following eligibility criteria.

\section{Patient inclusion criteria:}

Newly diagnosed non-metastatic localized resectable disease, primary OS, histological evidence of high-grade OS of an extremity (femur, tibia, humerus, fibula, radius, and ulna), age $\leq 18$ years at the date of the diagnostic biopsy, started chemotherapy within 30 days of diagnostic biopsy, and adequate bone marrow function; neutrophils count $>1.5 \mathrm{x}$ $10^{9} / \mathrm{L}$ ( or WBC $>3 \times 10^{9} / \mathrm{L}$ if neutrophils were not available) and platelet count $>100 \times 10^{9} / \mathrm{L}$, , renal function (glomerular filtration rate $\geq 70 \mathrm{ml} / \mathrm{min} / 1.73 \mathrm{~m}^{2}$ ), and liver function (total bilirubin $\leq 1.5 \times$ upper limit of normal (ULN)).

\section{Patient exclusion criteria:}

Any previous treatment for OS and any medical history of chemotherapy, juxtacortical (periosteal, parosteal) OS, unresectable primary disease, or metastatic, or both, and if patients had been administered any drug before MTX clinical protocol that can affect MTX level that would be recorded.

\section{Clinical protocol:}

All patients were treated with HDMTX $\left(12000 \mathrm{mg} / \mathrm{m}^{2} \mathrm{I} . \mathrm{V}\right.$ over 4-hours infusion), administered at weeks 4, 5, 9, and 10 as per protocol. The leucovorin rescue (LR) of $15 \mathrm{mg} /$ $\mathrm{m}^{2} / \mathrm{q}$ 6-hours started 24-hours after the initiation of the infusion of HDMTX..$^{13}$ The patients' data were collected from Cerner (electronic medical records) using (RED-Cap ${ }^{\circledR}$ ), a web-based data collection tool. 


\section{Genotyping:}

Blood samples $(5 \mathrm{ml})$ in EDTA vacutainers were collected for genotyping. Genomic DNA was extracted using the GeneJET $^{\text {TM }}$ kits TaqMan ${ }^{\circledR}$ - Thermo- fisher scientific Baltics UAB $\mathrm{V}$, Lithuania. DNA concentrations were detected using Nano-Quant Infinite M200 PRO (Tecan Group Ltd., Switzerland). Genotyping was carried out using Quant-Studio $12 \mathrm{~K}$ Flex Real-Time PCR System Life Technologies Ltd (Paisley PA4 9RF, UK). The predesigned and drug-metabolizing enzymes (DME) studied genes SNPs were processed according to (Thermo- fisher scientific Baltics protocol). The volumes of plate wells didn't exceed $10-\mu \mathrm{L}$ for the cycling system. Life Technologies software was adapted for allele discrimination analysis. ${ }^{12}$

\section{Plasma level analysis of MTX:}

The analyses of MTX levels were carried out post-24-hours infusion initiation time, and every 24-hours, until the MTX level was $\leq 0.2 \mu \mathrm{Mol} / \mathrm{L} .{ }^{14}$ This was measured by the enzyme multiple immunoassay techniques (EMIT ${ }^{\circledR}$ MTX 2011), supplied with Siemens Healthcare Diagnostics Inc., SYVA ${ }^{\circledR}$, USA. $^{13}$

\section{Outcome analysis:}

TN\% followed preoperative chemotherapy was histopathological determined. All patients were assessed for HDMTX toxicities and were graded and recorded according to the common terminology criteria for adverse events version 5 (CTCAE v5.0). 5-year overall survival (O.S) and event-free survival (EFS) were analyzed.

\section{STATISTICS}

The patients were divided into two groups according to genotyping results: group 1 was "homozygous wild", and group 2 included patients with at least one variant allele "homozygous variant and heterozygous". Patients' levels of MTX at 72-hour were categorized to "good elimination" (72-hour
$<0.2 \mu \mathrm{Mol} / \mathrm{L}$ ) or "delayed elimination" (72-hour $\geq 0.2$ $\mu \mathrm{Mol} / \mathrm{L}){ }^{15}$ To account for the correlated nature of the cumulative data, the generalized linear mixed model (GzLMM) fit by maximum likelihood (Laplace Approximation) for binomial data was used to test the association between SNPs and patterns of MTX plasma levels at 72-hour. The retrospectively measured toxicities for patients were divided into two types moderate (grade 1-2) and severe (grade 3-4). It followed CTCAE v5.0 guidelines for haematological toxicity, hepatotoxicity, and nephrotoxicity. TN, if $\leq 90 \%$ was considered to be poor responders as having more than $10 \%$ viable tumour at the time of surgery, and $>90 \%$ was considered to be good responders. ${ }^{16}$ Cumulative logit link function for ordinal outcomes was used to examine the relationship between SNPs and toxicities to determine the significance of the selected variables and to estimate effect sizes. 5-years O.S and EFS were estimated using Kaplan - Meier. The logrank test analyzed the study differences in the estimates of endpoint events over time concerning the SNPs investigated. Hazard ratios and $95 \%$ confidence intervals (CIs) were calculated by Cox proportional hazards regression model for univariate and multivariate analyses to identify independent predictors of O.S and EFS (both SNPs and demographic variables). Quantitative variables were summarized using the range, mean, and standard deviation. The frequency and percentage were used for qualitative variables. The analysis was done using $\mathrm{R}$ for statistical computing $\mathrm{R}$ version 3.6.1. The significance levels were adjusted using the Bonferroni method. The adjusted significance level was 0.0045 . Significance was considered at $p<0.004$.

\section{RESULTS}

\section{Patients' demographics}

One hundred and thirty-three patients were eligible and included in this study. The main characteristics of the patients were included in (Table 1).

\section{Table 1: The main characteristics of the patients $(\mathrm{N}=133)$}

$\begin{array}{lc}\text { Demographic data } & (\mathrm{N}=133) \\ \text { Gender } & \\ \text { Female } & 65(48.9 \%) \\ \text { Male } & 68(51.1 \%) \\ \text { Age }(\mathrm{y}) & \\ \text { Range } & 4-17.86 \\ \text { Mean } \pm \text { SD } & 12.51 \pm 3.33 \\ \left(B S A, \mathbf{m}^{2}\right) & \\ \text { Range } & 0.70-2.08 \\ \text { Mean } \pm \text { SD } & 1.40 \pm 0.31\end{array}$


Median

\section{Site of tumour}

Femur

Tibia

Fibula

Humerus

Radius

Ulna

\section{Outcome data of HDMTX chemotherapy}

(TN):

Poor responders $(\leq 90 \%)$

Good responders (>90\%)

Final status (Survival):

Alive

Dead

$$
\begin{gathered}
1.44 \\
(\mathrm{~N}=133) \\
77(57.8 \%) \\
44(33.1 \%) \\
6(4.5 \%) \\
4(3 \%) \\
1(0.75 \%) \\
1(0.75 \%)
\end{gathered}
$$

( $\mathrm{N}=126)$

$85(67.5 \%)$

$41(32.5 \%)$

( $\mathbf{N}=133)$

$106(79.9 \%)$

$25(18.8 \%)$

$2(1.5 \%)$

N: Number of patients, Y: year, SD: Standard deviation, BSA: Body surface area, HDMTX: High dose methotrexate, TN: Tumor necrosis.

\section{Genotyping:}

The results of genotyping and allelic frequencies are listed in (Table 2). The minor allele frequency (MAF) for $M T H-$ $F R$ (rs1801133) C> T, SLCO1B1 (rs4149081) A $>\mathrm{G}, A B C G 2$ (rs2231142) G> T, $A B C C 2$ (rs717620) $C>T$, and $A B C C 3$

\begin{tabular}{|c|c|c|c|c|c|c|}
\hline SNPs & genotyping & Alleles & Genotyping & Allele frequency & $\begin{array}{l}\text { Patients (Group 1) } \\
\text { frequency } \%\end{array}$ & $\begin{array}{l}\text { Patients (Group } \\
\text { 2) frequency } \%\end{array}$ \\
\hline & & & $\mathbf{N}$ & & (HW) & (HV and HT) \\
\hline
\end{tabular}
(rs4148412) $\mathrm{T}>\mathrm{C}$ were $(0.29,0.49,0.27,0.11$, and 0.42 ,

\begin{tabular}{|c|c|c|c|c|c|c|c|c|}
\hline MTHFR & $\mathrm{HW}^{*}$ & $\mathrm{CC}$ & 66 & 50.0 & \multirow{3}{*}{$\mathrm{C}=0.71$} & \multirow{3}{*}{$\mathrm{T}=0.29$} & \multirow{3}{*}{50} & \multirow{3}{*}{50} \\
\hline $\begin{array}{l}\text { rs1801133 } \\
(C>T)\end{array}$ & $\mathrm{HV}^{*}$ & TT & 12 & 9.1 & & & & \\
\hline$(\mathrm{N}=132)$ & $\mathrm{HT}^{*}$ & $\mathrm{CT}$ & 54 & 40.9 & & & & \\
\hline \multicolumn{9}{|c|}{ 2-SLCO1B1: } \\
\hline rs11045879 & HW & CC & 32 & 24.2 & \multirow{3}{*}{$\mathrm{C}=0.37$} & \multirow{3}{*}{$\mathrm{T}=0.63^{*}$} & \multirow{3}{*}{24.2} & \multirow{3}{*}{75.8} \\
\hline$(\mathrm{C}>\mathrm{T})$ & $\mathrm{HV}$ & $\mathrm{TT}$ & 66 & 50.0 & & & & \\
\hline$(\mathrm{N}=132)$ & HT & $\mathrm{CT}$ & 34 & 25.8 & & & & \\
\hline rS4149o81 & HW & $\mathrm{AA}$ & 43 & 33.1 & \multirow{3}{*}{$A=0.51$} & \multirow{3}{*}{$\mathbf{G}=0.49$} & \multirow{3}{*}{33.1} & \multirow{3}{*}{66.9} \\
\hline$(A>G$ & $\mathrm{HV}$ & GG & 42 & 32.3 & & & & \\
\hline$(\mathrm{N}=130)$ & HT & AG & 45 & 34.6 & & & & \\
\hline rS2306283 & HW & AA & 27 & 20.5 & \multirow{3}{*}{$\mathrm{A}=0.47$} & \multirow{3}{*}{$\mathbf{G}=0.53^{*}$} & \multirow{3}{*}{20.5} & \multirow{3}{*}{$79 \cdot 5$} \\
\hline$(A>G)$ & $\mathrm{HV}$ & GG & 35 & 26.5 & & & & \\
\hline$(\mathrm{N}=132)$ & HT & AG & 70 & 53.0 & & & & \\
\hline 3-ABCG2 & HW & GG & 69 & 51.9 & \multirow{3}{*}{$\mathbf{G}=0.73$} & \multirow{3}{*}{$\mathrm{T}=0.27$} & \multirow{3}{*}{51.9} & \multirow{3}{*}{48.1} \\
\hline rS2231142 & $\mathrm{HV}$ & $\mathrm{TT}$ & 7 & 5.2 & & & & \\
\hline $\begin{array}{l}(G>T) \\
(N=133)\end{array}$ & HT & GT & 57 & 42.9 & & & & \\
\hline
\end{tabular}

respectively). While for $S L C O 1 B 1$ (rs11045879) C $>\mathrm{T}, S L$ CO1B1 (rs2306283) $\mathrm{A}>\mathrm{G}, A B C C 3$ (rs 4793665) $\mathrm{C}>\mathrm{T}$, and $A B C C 3$ (rs 733392) $\mathrm{G}>\mathrm{A}$, the variant allele was more predominant with an allelic frequency of $(0.63,0.53,0.60$, and 0.85 , respectively).

Table 2: Genotypes frequencies and allele frequency of the studied genes SNPs 


\begin{tabular}{|c|c|c|c|c|c|c|c|c|}
\hline 4-ABCC2 & HW & $\mathrm{CC}$ & 107 & 80.5 & \multirow{3}{*}{$\mathrm{C}=0.89$} & \multirow{3}{*}{$\mathrm{T}=0.11$} & \multirow{3}{*}{80.5} & \multirow{3}{*}{19.5} \\
\hline \multirow{2}{*}{$\begin{array}{l}\operatorname{rs717620}(C>T) \\
(N=133)\end{array}$} & $\mathrm{HV}$ & TT & 2 & 1.5 & & & & \\
\hline & HT & $\mathrm{CT}$ & 24 & 18.0 & & & & \\
\hline \multicolumn{9}{|l|}{ 5-ABCC $3:$} \\
\hline \multirow{3}{*}{$\begin{array}{l}\text { rs4148412 } \\
(\mathrm{T}>\mathrm{C}) \\
(\mathrm{N}=130)\end{array}$} & HW & TT & 49 & $37 \cdot 7$ & \multirow{4}{*}{$\mathbf{T}=0.58$} & \multirow{4}{*}{$\mathrm{C}=0.42$} & \multirow{4}{*}{$37 \cdot 7$} & \multirow{3}{*}{62.3} \\
\hline & $\mathrm{HV}$ & $\mathrm{CC}$ & 27 & 20.8 & & & & \\
\hline & HT & $\mathrm{TC}$ & 54 & 41.5 & & & & \\
\hline rs $479366_{5}$ & HW & $\mathrm{CC}$ & 18 & 14.0 & & & & \\
\hline$(C>T)$ & $\mathrm{HV}$ & TT & 43 & $33 \cdot 3$ & \multirow[t]{2}{*}{$\mathrm{C}=0.40$} & \multirow[t]{2}{*}{$\mathrm{T}=0.60^{*}$} & \multirow[t]{2}{*}{14.0} & \multirow[t]{2}{*}{86.0} \\
\hline$(\mathrm{N}=129)$ & HT & $\mathrm{CT}$ & 68 & 52.7 & & & & \\
\hline rs733392 & HW & GG & 2 & 1.5 & \multirow{3}{*}{$\mathbf{G}=0.15$} & \multirow{3}{*}{$\mathrm{A}=0.85^{*}$} & \multirow{3}{*}{1.5} & \multirow{3}{*}{98.5} \\
\hline$(\mathbf{G}>\mathbf{A})$ & HV & $\mathrm{AA}$ & 94 & 72.3 & & & & \\
\hline$(\mathrm{N}=130)$ & HT & GA & 34 & 26.2 & & & & \\
\hline
\end{tabular}

*Predominant variant alleles, HW: Homozygous wild genotype; HT: Heterozygous genotype; HV: Homozygous variant genotype., SNPs: Single nucleotide polymorphisms, rs: reference SNP number, N: Number of patients, A: Adenine, G: Guanine, C: Cytosine, T: Thymine, MTHFR: Methylene-tetrahydrofolate reductase, SLCO1B1: Solute Carrier Organic Anion Transporter Family Member 1B1, ABCG2: ATP Binding Cassette Subfamily G Member 2, ABCC2: ATP Binding Cassette Subfamily C Member 2, ABCC 3 : ATP Binding Cassette Subfamily C Member 3.

\section{Genotypes-MTX level analysis}

Association between the two patients' groups of each gene SNP and delayed MTX pattern at 72-hour MTX levels is illustrated in (Table 3). Only age was significantly associated with delayed elimination at 72-hour. A one-year increase in patients' age was associated with a $19 \%$ increase in the risk of MTX delayed excretion $[p=0.00059$, OR $1.19,95 \%$ CI $(1.09-1.29)]$.

Table 3: Association between the two patient groups of each SNP and delayed elimination at 72-hour after HDMTX infusions

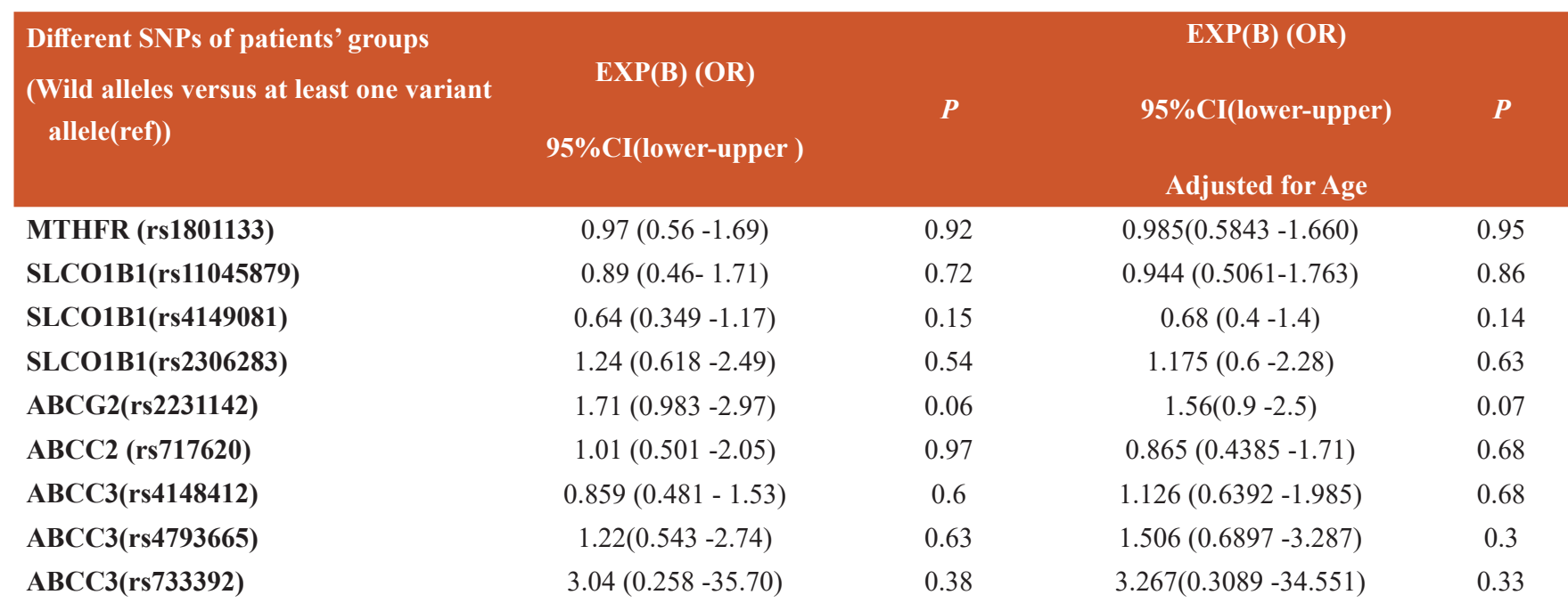

Gender

Female(ref)

0.18

Male

$1.47(0.84-2.55)$

Age 1.19(1.09- 1.29)

0.00059*

*Significant $(\mathrm{P} \leq \mathrm{0.0045}), \operatorname{Exp}(\mathrm{B})=$ Estimated Odds Ratio (OR), CI: The confidence interval, (ref): Reference group, SNPs: Single nucleotide polymorphisms, rs: reference SNP number, MTHFR: Methylene-tetrahydrofolate reductase, SLCO1B1: Solute Carrier Organic Anion Transporter Family Member 1B1, ABCG2: ATP Binding Cassette Subfamily G Member 2, ABCC2: ATP Binding Cassette Subfamily C Member 2, ABCC3: ATP Binding Cassette Subfamily C Member 3. 


\section{Genotypes-toxicity analysis}

Older patients were associated with protection of developing higher grades of enzymatic hepatotoxicity for (ALT) $[p$ $=0.002$, OR 0.88, 95\% CI (0.8-0.95) $]$ and (AST) $[p=0.001$, OR $0.82,95 \%$ CI (0.76-0.89)]. Patients with $(C C)$ genotypegroup of $A B C C 3$ (rs 4793665) $\mathrm{C}>\mathrm{T}(14 \%$ of the studied population) associated with protective effect against severe nephrotoxicity $[p=0.003$, OR $0.31,95 \%$ CI $(0.14-0.67)]$. Association between the two patients' groups of each gene SNP, age, gender, and different severe toxicities after four HDMTX cycles is illustrated in (Table 4).

Table 4: Association between the two patient groups of each SNP and different severe toxicities after four HDMTX cycles

\begin{tabular}{|c|}
\hline $\begin{array}{l}\text { Different SNPs of pa } \\
\text { (wild alleles versus a }\end{array}$ \\
\hline MTHFR (rs1801133) \\
\hline SLCO1B1(rs11045879) \\
\hline SLCO1B1(rs4149o81) \\
\hline SLCO1B1(rS2306283) \\
\hline $\mathrm{ABCG}_{2}(\mathrm{rs2231142)}$ \\
\hline $\mathrm{ABCC}_{2}$ (rs717620) \\
\hline $\mathrm{ABCC}_{3}(\mathrm{rs} 4148412)$ \\
\hline $\mathrm{ABCC}_{3}\left(\mathrm{rs}_{4793665)}\right.$ \\
\hline $\mathrm{ABCC}_{3}($ rs733392) \\
\hline Gender \\
\hline Age \\
\hline
\end{tabular}

MTHFR (rs1801133)

SLCO1B1(rs11045879)

SLCO1B1(rs4149081)

SLCO1B1(rs2306283)

ABCG2(rs2231142)

$\mathrm{ABCC}_{2}$ (rs717620)

$\mathrm{ABCC}_{3}$ (rs4148412)

$\mathrm{ABCC}_{3}$ (rs4793665)

$\mathrm{ABCC}_{3}$ (rs733392)

Gender

Age

SLCO1B1(rs11045879)

SLCO1B1(rs4149081)

SLCO1B1(rs2306283)

ABCG2(rs2231142)

$\mathrm{ABCC}_{2}$ (rs717620)

$\mathrm{ABCC}_{3}$ (rs4148412)

$\mathrm{ABCC}_{3}$ (rs4793665)

$\mathrm{ABCC}_{3}$ (rs733392)

Age

Gender

Female (ref)

\section{$\operatorname{Exp}(B)(95 \%$ CI} for $\operatorname{Exp}(B)$

Hepatotoxicity (ALT)

$\begin{array}{lc}0.73(0.42-1.26) & 0.26 \\ 0.63(0.3-1.19) & 0.15 \\ 1.29(0.72-2.31) & 0.39 \\ 0.99(0.51-1.98) & 0.99 \\ 1.13(0.66-1.95) & 0.66 \\ 0.90(0.45-1.78) & 0.75 \\ 1.01(0.57-1.79) & 0.97 \\ 1.23(0.55-2.76) & 0.61 \\ 1.30(0.14-11.9) & 0.82 \\ 0.93(0.54-1.61) & 0.79 \\ 0.88(0.8-0.95) & \mathbf{0 . 0 0 2}\end{array}$

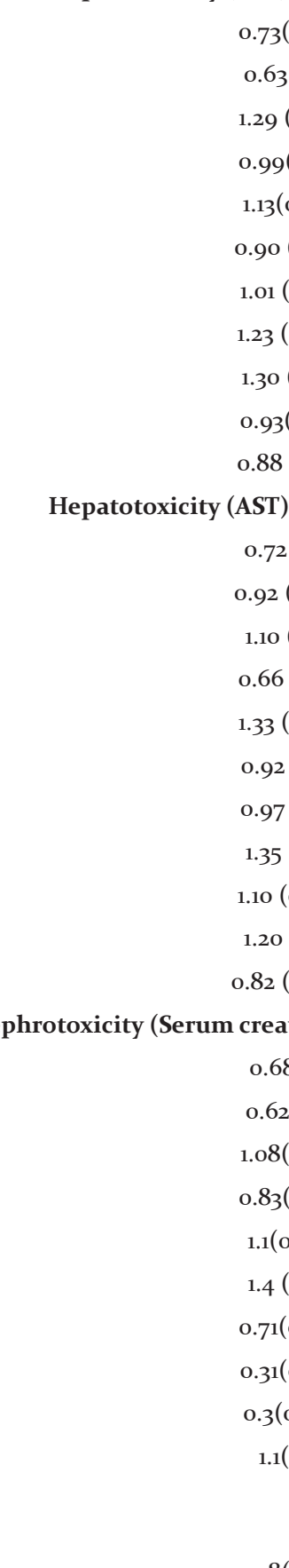

$\begin{array}{cc}0.72(0.41-1.3) & 0.29 \\ 0.92(0.48-1.79) & 0.81 \\ 1.10(0.58-1.9) & 0.84 \\ 0.66(0.33-1.31) & 0.23 \\ 1.33(0.77-2.33) & 0.31 \\ 0.92(0.45-1.8) & 0.80 \\ 0.97(0.6-1.42) & 0.94 \\ 1.35(0.59-3.1) & 0.48 \\ 1.10(0.25-4.62) & 0.92 \\ 1.20(0.65-2.1) & 0.60 \\ 0.82(0.76-0.89) & 0.001^{*}\end{array}$

Nephrotoxicity (Serum creatinine)

$\begin{array}{cc}0.68(0.4-1.2) & 0.15 \\ 0.62(0.33-1.2) & 0.14 \\ 1.08(0.72-1.62) & 0.7 \\ 0.83(0.43-1.62) & 0.59 \\ 1.1(0.66-1.89) & 0.69 \\ 1.4(0.7-2.62) & 0.35 \\ 0.71(0.48-1.06) & 0.09 \\ 0.31(0.14-0.67) & 0.003^{*} \\ 0.3(0.06-1.44) & 0.13 \\ 1.1(1.03-1.2) & 0.009\end{array}$

$0.8(0.471 .36)$

$0.72(0.41-1.3) \quad 0.29$

0.81

0.84

0.23

0.31

80

0.94

0.48

0.92

$.001^{*}$

Male

*Significant $(P \leq 0.0045)$, Exp (B): Estimated Odds Ratio (OR), CI: The confidence interval, ALT: alanine transaminase, AST: aspartate aminotransferase, (ref): Reference group, SNPs: Single nucleotide polymorphisms, rs: reference SNP number, MTHFR: Methylene-tetrahydrofolate reductase, SLCOıB1: Solute Carrier Organic Anion Transporter Family Member 1B1, ABCG2: ATP Binding Cassette Subfamily G Member 2, ABCC2: ATP Binding Cassette Subfamily C Member 2, ABCC 3: ATP Binding Cassette Subfamily C Member 3. 


\section{Genotypes-outcome analysis}

The SNPs had not been significantly associated with TN\% or O.S and EFS. The survival analysis for $A B C C 3$ (rs4793665) $\mathrm{C}>\mathrm{T}$ is illustrated in (Figures $1 \mathrm{a}$ and1b).

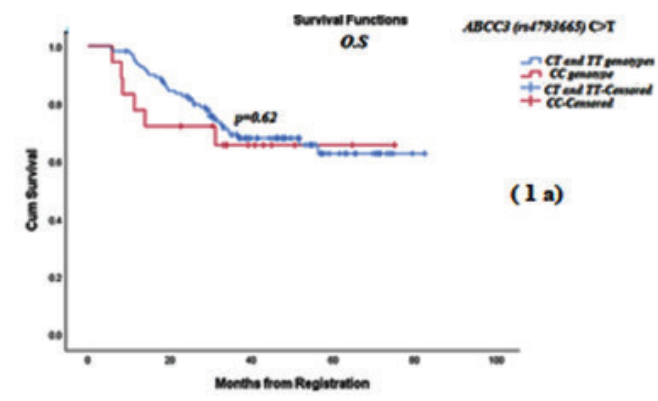

Figure1a: The Kaplan - Meier (5years O.S) for ABCC3 rs4793665 C>T

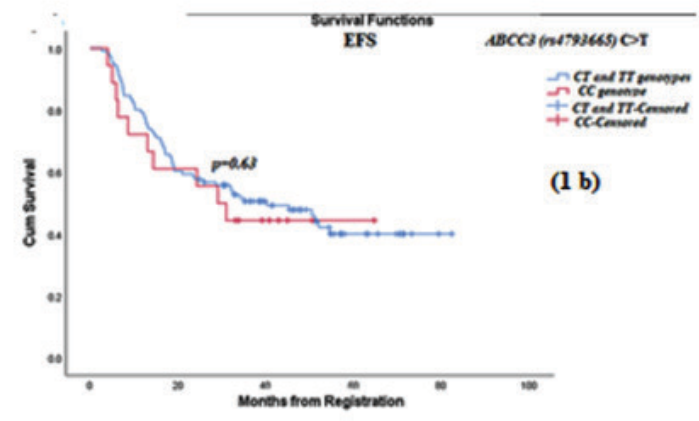

Figure 1b: The Kaplan - Meier (5years EFS) for ABCC3 rs4793665 C>T.

\section{DISCUSSION}

This study examined the emerging role of the pharmacogenetic markers in children with OS to minimize inter-individual variations in chemotherapy such as HDMTX. The objectives of many studies are to design prognostic tools for protection against any potential toxicity resulting in treatment failure, prolongation of hospitalization, and decrease in survival. It was achieved by studying the relation between PGs involving gene SNPs, drug PKs, and PDs. The problem here, many studies had divergent, conflicting findings and a lack of reproducibility, a small number of homogeneous ethnicities, variations in ethnic groups, differences in treatment protocols, varied assessment of toxicity criteria, and clinical outcomes. ${ }^{17}$ The present study offered essential findings on a retrospective cohort of patients larger than published in prior similar studies. It provided an in-depth analysis of a homogeneous Egyptian ethnicity with the same pre-therapy conditions. They had been treated with the same protocol, reliable, and well-recorded data using the Bonferroni correction method. Toxicity criteria were available/used, survival, and TN\% analyses were performed.
The most important clinically relevant finding was the reliability of the MTX terminal elimination pattern at 72-hour. The relationship is based on the studies of Imanishi and Trevino et al. on HDMTX in pediatric acute lymphoblastic leukaemia (ALL). ${ }^{8}$ Therefore, our study relied on an objective and quantifiable marker that was cut off the 72-hour MTX level. Regarding the allelic frequency determination, the significant finding was that the variant allele was more predominant with an allelic frequency of SLCO1B1 (rs 11045879) $C>T, \quad S L C O 1 B 1 \quad(r s 2306283) \quad A>G, A B C C 3$ (rs4793665) $C>T$, and $A B C C 3$ (rs 733392) $G>A$ in Egyptian children with OS. Recent developments in the field of PGs have led to a renewed interest in the polymorphic effect of transporter and metabolism genes on PKs and PDs of MTX.

In the current study, a one-year increase in age was associated with a $19 \%$ increased risk of MTX delayed elimination pattern at 72-hour. So, older age children were considered poor eliminators of HDMTX in the studied sample. The interpretation of our results may be the low volume of distribution of MTX. The young children have high body water content leading to more MTX elimination than elder children. These results match those observed in earlier studies. ${ }^{18,19}$ The study of Csordas et al. reported the significant association between high MTX levels in children aged more than 14 years because the metabolism resembles those of adult patients. The younger children were less likely to have delayed elimination due to their physiology. ${ }^{18}$ The findings of the present study further support the idea of different genotypes did not have a significant association with MTX level as consistent with prior studies. ${ }^{13,18}$

Prior studies demonstrated that the risk factors related to the appearance of certain types of toxicities after HDMTX administration. These factors may be due to higher MTX levels after multiple HDMTX cycles or other factors. The previous studies offered contradictory findings of several factors that participate in this association. ${ }^{19-21}$ The high MTX level wouldn't predict the appearance- a specific type of toxicity except for nephrotoxicity after multiple HDMTX cycles, described by Tsurusawa et al. ${ }^{22}$ Mandal et al. revealed that MTX levels didn't associate with toxicity. He explained that gender, body mass index, and the pre-cycles ANC made that association. Besides, basic serum creatinine was the predictor of toxicity. ${ }^{20}$ The study of Vaishnavi et al. in low and middle-income countries at a single centre in (Chandigarh, India) recommended that the safe give full HDMTX to ALL patients with extended hydration, additional doses of LR, monitoring of serum creatinine, and $\mathrm{pH}$ without therapeutic drug monitoring (TDM) of MTX. ${ }^{23}$

Herein, older children have significant protection against the risk of severe elevation in liver enzymes, consistent with Holmboe et al. ${ }^{24}$ The increase in (ALT) and (AST) values might be temporary and reversible, which may not result in 
chronic liver disease following administration of HDMTX. ${ }^{25}$ Our study's recommendations are to translate our findings to patients' bedsides as preventive measures. AST and ALT levels should be closely monitored before and during MTX cycles. Concurrent hepatic inflammation or dysfunction exacerbated by HDMTX doses should be managed, particularly in younger children.

As far as $A B C C 3$ is located in the gall bladder, and kidney. The expression of $A B C C 3$ mRNA is associated with drug resistance. Its biological function is organic anion excretion like MTX and bile. ${ }^{26}$ Children in group 1 having $A B C C 3$ (rs4793665) (CC) genotype (14\% of the tested population) were associated with protection against the risk of severe nephrotoxicity even after adjusting for age. The findings were inconsistent with prior studies. ${ }^{6,12}$ The study results of Hegyi et al. demonstrated that the presence of at least one variant allele $\mathrm{T}$ in patients with $\mathrm{ABCC} 3$ (rs4793665) causes higher MTX concentrations than wild-type allele C. ${ }^{6}$ Consequently, our findings explain children with allele $\mathrm{C}$ may cause protection against impaired excretion of MTX, 7-hydroxy-MTX, and inactive 7-hydroxy-MTX, and 4-amino-4-deoxy-Nmethylpteroic acid (DAMPA). The high-risk patients who suffered from severe nephrotoxicity had involved in the solubilities of HDMTX metabolites in urine may reach ten folds lower than MTX. As a consequence, it may cause renal tubules obstruction, and extended contact of MTX with the renal tubular epithelial tissue lining exposes it to direct toxic AKI. ${ }^{27}$ Even after subsequent LR doses as it cannot reverse nephrotoxicity, so patients in group 1 (CC) genotype have high protective mechanisms against the risk of irreversible acute kidney failure. They are expected to be more protected and susceptible to resist the increase in their serum creatinine by more than $50 \%$ within 24 -hours. ${ }^{21,2825}$ The strong point in our study has a large sample size that proved the toxicity associated with SNPs, as consistent with recommendations of Derotte et al. ${ }^{12}$ Our study proved that the nephrotoxicity risk could be prevented by $A B C C 3$ (rs4793665) C $>$ T screening.

Herein, our study offered some essential insights into the absence of the significant association of TN\% with different gene SNPs, which were consistent with previous publications like Serra et al. ${ }^{29}$ Eventually, the study has gone some way towards enhancing our understanding of the clinical outcome of survival analysis by The Kaplan - Meier estimation for the studied SNPs. The observed difference between O.S and EFS with SNPs genotypes in this study hadn't reached a significant level. Further work is required to establish this association. In the present study, there are some notable limitations to be noted. The possibility of having other unknown factors affects PKs and PDs of HDMTX. Our findings are limited to our hospital centre, as the analysis had relied on a single centre.

\section{CONCLUSION}

To sum up, our study provided a preventive measure of toxicity that might associate with HDMTX in Egyptian children with OS. Factors and candidate biomarkers could affect the toxicity and HDMTX elimination by the concurrent analysis of PGs and other factors like age. For the future best clinical practice, the present study recommended that: Firstly, screening patients for $A B C C 3$ (rs4793665) SNP: 14\% of Egyptian children in the study with (CC) genotype were protected against the risk of irreversible severe nephrotoxicity. Secondly, more genetic investigations are needed for MTX dose adjustment and protection against hazards of HDMTXinduced nephrotoxicity in children with OS.

\section{ACKNOWLEDGEMENTS}

We thank the patients for kindly supplying biological samples in CCHE-57357. We thank the laboratory of biorepository and specimen laboratory department at CCHE-57357, Cairo, Egypt for laboratory work.

\section{Authors' contributions:}

AZ, RL, EE: Participated in the design of the study and approval of the final version of the manuscript. RL, SA, AZ: Interpretation of laboratory results. $\mathrm{OH}$ : Statistical analysis of data. AZ, RL: Implementation of laboratory investigation and acquisition of data. EE: Clinical data. AS: Clinical pathology. The authors have read and approved the manuscript. Funding: [AZ: (6 SNPs)] and [RL: (3 SNPs)]

\section{Source of funding: Self}

\section{Conflict of interest: Nil}

\section{DECLARATIONS}

\section{Ethics approval and consent to participate:}

The study was approved by the Institutional Review Board with serial number 1112018 (25 June 2018) at CCHE-57357. All participants in the original trials or their parent/legal guardian signed a written document of informed consent and assent was obtained as appropriate.

\section{Consent for publication:}

Available on request.

\section{Availability of data and material:}

The datasets used and/or analyzed during the current study are available from the corresponding author on reasonable request.

\section{Competing interests:}

The authors declare that they have no conflicting interests. 


\section{REFERENCES}

1. Durfee, R.A., M. Mohammed, And H.H. Luu. Review Of Osteosarcoma And Current Management. Rheumatol Ther, 2016. 3(2): P. 221-243.

2. El-Refaey, O.M. Intensive Chemotherapy For Resectable HighGrade Osteosarcoma Of The Extremities. Cu Theses, 2016.

3. Tsuda, Y., Et Al. The Outcomes And Prognostic Factors In Patients With Osteosarcoma According To Age: A Japanese Nationwide Study With Focusing On The Age Differences. BMC Cancer, 2018. 18(1):1-7.

4. LuengoA., D.Y. Gui, M.G. Vander Heiden.Targeting Metabolism For Cancer Therapy. Cell Chem. Biol., 2017. 24(9):11611180.

5. Lui G. A Pharmacokinetic And Pharmacogenetic Analysis Of Osteosarcoma Patients Treated With High-Dose Methotrexate: Data From The OS2006/Sarcoma-09 Trial. J. Clin. Pharmacol, 2018. 58(12):1541-1549.

6. Hegyi, M. Pharmacogenetic Analysis Of High-Dose Methotrexate Treatment In Children With Osteosarcoma. Oncotarget, 2017. 8(6):9388.

7. Giletti AP. Esperon.Genetic Markers In Methotrexate Treatments. Pharmacogenomics J., 2018. 18(6): P. 689-703.

8. Treviño LR. Germline Genetic Variation In An Organic Anion Transporter Polypeptide Associated With Methotrexate Pharmacokinetics And Clinical Effects. J. Clin. Oncol., 2009. 27(35):5972.

9. HowardS.C.Preventing And Managing Toxicities Of High-Dose Methotrexate. The Oncologist, 2016. 21(12):1471-1482.

10. KalyanpadY. Methotrexate A Double-Edged Sword-Effect Of Methotrexate In Patients Of Psoriasis Due To Medication Errors. Int J Cur Res Rev, 2017. 9(20): P. 10.

11. Jiang, X.-L. Clinical Pharmacokinetics And Pharmacodynamics Of Clopidogrel. Clin. Pharmacokinet., 2015. 54(2): 147-166.

12. De ROTTE, M.C. ABCB1 And ABCC3 Gene Polymorphisms Are Associated With First-Year Response To Methotrexate In Juvenile Idiopathic Arthritis. J. Rheumatol., 2012. 39(10):20322040.

13. El Mesallamy, H.O.High-Dose Methotrexate In Egyptian Pediatric Acute Lymphoblastic Leukemia: The Impact Of ABCG2 C421A Genetic Polymorphism On Plasma Levels, What Is Next? J. Cancer Res. Clin. Oncol., 2014. 140(8):1359-1365.

14. 14. Howard SC. Preventing And Managing Toxicities Of High-Dose Methotrexate. The Oncologist, 2016. 21(12):1471.

15. Martinez D. Endogenous Metabolites-Mediated Communication Between OAT1/OAT3 And OATP1B1 May Explain The As- sociation Between SLCO1B1 Snps And Methotrexate Toxicity. Clin. Pharmacol. Ther., 2018. 104(4):687-698.

16. Hattinger CM. Pharmacogenomics Of Genes Involved In Antifolate Drug Response And Toxicity In Osteosarcoma. Expert Opinion On Expert Opin. Drug Metab. Toxicol., 2017. 13(3):245-257.

17. Lopez-Lopez E.Pharmacogenetics Of Childhood Acute Lymphoblastic Leukemia. Pharmacogenomics, 2014. 15(10):13831398.

18. Csordas, K. Comparison Of Pharmacokinetics And Toxicity After High-Dose Methotrexate Treatments In Children With Acute Lymphoblastic Leukemia. Anti-Cancer Drugs, 2013. 24(2):189197.

19. Young EP. Risk Factors Associated With Delayed Methotrexate Clearance And Increased Toxicity In Pediatric Patients With Osteosarcoma. Pediatr. Blood Cancer, 2020. 67(4): E28123.

20. Mandal P. Adverse Effects With Intravenous Methotrexate In Children With Acute Lymphoblastic Leukemia/Lymphoma: A Retrospective Study. Indian J Hematol Blood Transfus, 2020:17.

21. LiX. Identifying Risk Factors For High-Dose Methotrexate-Induced Toxicities In Children With Acute Lymphoblastic Leukemia. Cancer Manag. Res., 2019. 11: 6265.

22. TsurusawaM. Statistical Analysis Of Relation Between Plasma Methotrexate Concentration And Toxicity In High-Dose Methotrexate Therapy Of Childhood Nonhodgkin Lymphoma. Pediatr. Blood Cancer, 2015. 62(2):279-284.

23. Vaishnavi K. Improving The Safety Of High-Dose Methotrexate For Children With Hematologic Cancers In Settings Without Access To MTX Levels Using Extended Hydration And Additional Leucovorin. Pediatr. Blood Cancer, 2018. 65(12): E27241.

24. HolmboeL. High Dose Methotrexate Chemotherapy: Pharmacokinetics, Folate And Toxicity In Osteosarcoma Patients. Br. J. Clin. Pharmacol., 2012. 73(1): P. 106-114.

25. HowardS.C. Preventing And Managing Toxicities Of HighDose Methotrexate. The Oncologist. 2016; 20(10) 0160-0164.

26. Liu, S. Predictive Potential Of ABCB1, ABCC3, And GSTP1 Gene Polymorphisms On Osteosarcoma Survival After Chemotherapy. Tumour Biol. 2014. 35(10): 9897-9904.

27. PerazellaM.A.,G.W. Moeckel. Nephrotoxicity From Chemotherapeutic Agents: Clinical Manifestations, Pathobiology, And Prevention/Therapy. In Seminars In Nephrology. 2010. Elsevier.

28. SangeethaN. an invitro study of cisplatin induced nephrotoxicity in goat kidney.Int J Cur Res Rev , 2012. 4(06)

29. SerraM., C. Hattinger.The Pharmacogenomics Of Osteosarcoma. Pharmacogenomics J., 2017. 17(1): P. 11. 